\title{
Enhancing an Automated Braille Writing Tutor
}

\author{
M. Bernardine Dias, M. Freddie Dias, Sarah Belousov, \\ Mohammed Kaleemur Rahman, Saurabh Sanghvi, Noura El-Moughny \\ Carnegie Mellon University \\ Pittsburgh, PA, USA and Doha, Qatar \\ \{mbdias, mfdias, sarahtbw\}@cs.cmu.edu, \{kaleem, scsanghvi\}@cmu.edu, \\ noura.elmoughnydalumni.cmu.edu
}

\begin{abstract}
The reported work advances the state-of-the-art in assistive technology for the blind by enhancing a low-cost automated tutor designed to teach braille writing skills to visually impaired children using voice feedback. We first provide some background on how the methodology of Intelligent Tutoring Systems correlates to an automated tutor for teaching braille writing skills. We then build on prior work to enhance our automated Braille Writing Tutor in three dimensions: (1) Initial field testing in three different countries; (2) Exploring customization needs for improving relevance in different cultures; and (3) Adding relevant games for increasing motivation. The outcome of this work is an enhanced low-cost tool that can help to increase braille literacy in blind communities around the world.
\end{abstract}

\section{INTRODUCTION}

A LMOST $90 \%$ of the world's visually impaired people live in developing communities [3] and it is estimated that less than $10 \%$ of the blind children in these communities have access to formal education[2]. Braille is a widely-used language that is the only means of literacy for blind people. Despite its significance and the accessibility it brings, learning to write braille still has a number of barriers. The traditional method of writing braille itself creates formidable challenges to literacy [1]. Each braille character is formed using 6 dots placed in a cell of two columns and three rows as shown in Figure 1 (a). A subset of these 6 dots is embossed to represent each character. The positions of the dots are universally numbered from 1 to 6 in a standardized manner. In developed countries, braille is usually embossed with a 6-key typewriter known as a Brailler. These devices are fast and easy to use but usually cost around US\$650 each [4]. In developing countries, such devices are sometimes available in small quantities at schools but are prohibitively expensive and so braille is almost always written with a slate and stylus as shown in Figure 1 (b). Using these tools, braille is typically written from right to left so that the page can be read from left to right when it is

This research was supported in part by the Qatar National Research Fund's National Priorities Research Program (NPRP) through grant \#30-6-7-91, the Qatar National Research Fund's Undergraduate Research Experience Program (UREP) through grant \#4-15-6, the Qatar Foundation for Education, Science, and Community Development, and discretionary gifts to the TechBridgeWorld research group at Carnegie Mellon University. The opinions expressed in this paper are those of the authors and do not necessarily represent the views of the sponsors. removed from the slate and turned over. Thus, learning to write braille can be difficult for the following reasons. First, children must learn mirror images of all letters, which doubles the alphabet and creates a disparity between the written and read forms of each letter. Second, feedback is delayed until the paper is removed and then flipped over and read. For young children, this delay can make braille conceptually challenging because the act of writing has no immediate feedback. It also takes longer for both the student and the teacher to identify and correct mistakes, and this slows the learning process. Together, these challenges contribute to the problem of illiteracy among the blind; especially in developing communities. Even in developed countries, writing braille with a slate and stylus is still viewed as the simplest and most affordable and portable format for note taking. In fact, some efforts are being made in the USA to renew the interest and skills for writing braille using a slate and stylus. Thus, assistive braille writing technology can have significant impact on millions of lives.

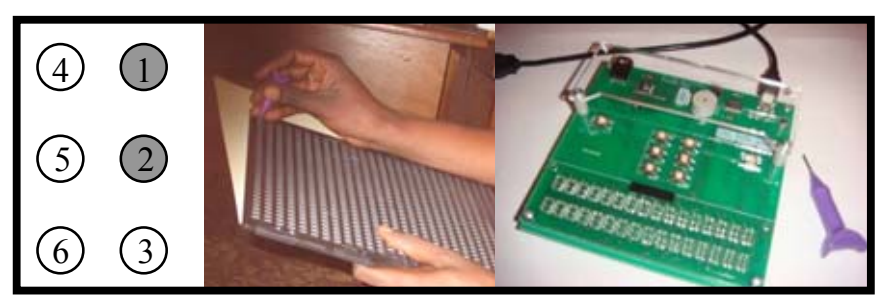

(a)

(b)

(c)

Figure 1: (a) Numbered braille cell with written English braille letter "B" (embossed dots are shown shaded); (b) Regular Slate and Stylus;

(c) Automated Braille Writing Tutor

We address some of these challenges in learning to write braille through the automated tutor shown in Figure 1 (c). This paper reports several enhancements to this tutor. We first explore how the methodology of Intelligent Tutoring Systems (ITS) correlates to an automated tutor for teaching braille writing skills. We then provide background information on the Braille Writing Tutor (BWT) we developed. Next we describe key enhancements we made to this tutor and discuss feedback we received from field testing this device. We conclude by summarizing our contributions and outlining directions for future work. 


\section{DESIGNING A BRAILLE WRITING TUTOR}

We first explore how this work can benefit from the literature on Intelligent Tutoring Systems (ITS) [6]. ITSs are interactive learning environments based on instructions and guided practice assisted by computers. They offer the ability to present educational materials through a communication interface to the computer and respond intelligently and instructively to the student's performance. The intelligent response of the tutor is adapted to a particular student through a process described in three phases: (1) Getting the information from the student; (2) Processing the information to update a model of the student; (3) Using the student model to provide customized feedback.

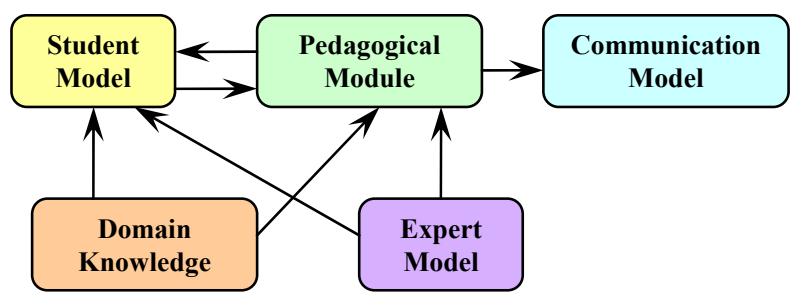

Figure 2: ITS components and their interactions [6]

The core components of an ITS and their interactions are shown in Figure 2. The Domain Knowledge contains the information being taught by the tutor. The Pedagogical Module captures the teaching process and makes decisions about how to guide the student. The Expert Model captures the skills of an expert of the knowledge stored in the Domain Knowledge and acts as a benchmark to evaluate the student performance. The Communication Model defines the interaction between the student and the system. Finally, the Student Model gathers and stores information specific to the current student, tracks the progress of the student based on the model, and provides useful data to the Pedagogical Module. Next, we briefly explore the mapping of each of these components to an ITS for teaching braille writing.

\section{A. Domain Knowledge}

For the purpose of teaching braille, this component will contain information about the numbering of the 6 dots, and the character mappings from the alphabet of a given braille language to the correct combination of dots in a braille cell. While this module is simple for basic braille, this module will need to include rules for contractions, special symbols, and operators (for use in mathematics and languages that include accents) for teaching more advanced braille.

\section{B. Pedagogical Module}

The pedagogical module is derived from the teacher's role in the classroom. It guides in the form of one-on-one interactions to maintain the individualized attention provided by an ITS. Two forms of the individualized instructions are used in an ITS; meta-strategies and instructional strategies [7]. Meta-strategies refer to the overall picture of the teaching process and include earning students' trust, covering the material in the curriculum, and assessing the students' ability to continue to the next grade level through a final assessment process. Instructional strategies refer to the methods used to teach a particular concept, including daily classroom activities and continuous assessment [8].

\section{Expert Model}

A benchmark for comparison and evaluation of the student's solutions is provided by the expert model. Two approaches for implementing this component are: (1) A rulebased approach where a set of rules are applied to solve different problems; (2) A scenario-based approach where the expert specifies how the student should respond in different scenarios [6]. Since the process of writing braille is based on a set of rules, a rule-based approach is best for this task.

\section{Communication Model}

The communication model addresses the question of how to present material and receive feedback from the student in the most effective manner. While most ITSs use graphical interfaces and visual cues to communicate with students, visual feedback is inaccessible to blind students and therefore is inappropriate for teaching braille. Hence, we must rely primarily on audio and tactile feedback for the student. The major drawback of audio feedback is that it prevents the use of the tutor by individuals who are both blind and deaf. Further, deciding what type of audio is best will depend upon the student's age, culture, and level of progress. Tactile communication on the other hand is often more complex and more expensive to incorporate.

\section{E. Student Model}

The student model is crucial to any ITS because it allows the tutor to adaptively provide customized feedback to each student. Common approaches to building a Student Model include Bayes Nets [9], the Overlay approach[14], and the Stereotype approach [10].

\section{BRAILLE WRITING TUTOR BACKGROUND}

We now briefly describe some background information on the BWT developed in our prior work [13]. The BWT is designed to provide guided practice using audio feedback for young children learning to write braille. It consists of an electronic slate (E-slate) and a stylus, which monitors the student's writing and transmits data in real time to a computer for immediate audio feedback. The latest version of the BWT consists of two rows of 16 braille cells and 6 buttons that work as tactile inputs. The stylus is a standard braille stylus that connects to the E-slate by its metal tip. Students can also use the two control buttons placed on the sides of the E-slate to perform a variety of mode changes.

The current implementation of the BWT focuses on basic braille. Therefore the domain knowledge component includes numbering of the 6 dots and the basic character maps for several languages including English and Arabic. 
The pedagogical module is somewhat more complex. First, the BWT earns a student's trust through a variety of strategies such as recording a teacher's voice for the audio feedback (young blind students often base their trust on familiar voices), reducing the number of wires (students are taught to stay away from wires), and using "happy sounds" to eliminate any fears of using a new device. We also improve the trust in the tutor's ability to teach by getting a respected person (such as a teacher) to endorse the tutor and introduce it to the student. The curriculum covered by the BWT was developed based on methods of teaching used in several schools for the visually impaired and include modes for dot practice, letter practice, and spelling. Assessment in the BWT is handled through quizzes that assess the two main aspects of writing braille; accuracy and speed. The solutions to the exercises in the BWT only require knowledge of the dot numbering and patterns, and therefore the expert model is easily implemented. In terms of the communication model, the buttons and stylus provide a tactile input interface to the tutor, while a set of pre-recorded audio files form the output from the BWT. Finally, the BWT uses a simple knowledge tracing algorithm to track student progress and provide some adaptive feedback [5].

\section{ENHANCING THE BRAILLE WRITING TUTOR}

Further details of the BWT are discussed in prior publications ([1], [5]). We build on this prior work in several ways. These enhancements, which are described next, are the basis of the work reported in this paper.

\section{A. Additional Feedback}

A key limitation of the previous work was that the BWT was only field tested in one school in India. To ascertain the potential impact of the BWT in other locations, we expanded our set of pilot tests to two other locations, and also returned to the original school to get feedback on the enhanced tutor. Our primary goals through these pilot tests were to get initial feedback on how well the BWT (1) complements classroom instruction, (2) is customizable for different cultures, and (3) motivates students to learn braille. To this end, we conducted pilot field-studies in schools in three countries: India, Qatar, and Zambia. Figure 3 and Figure 4 show the field tests in two of these locations.

The field work in India was facilitated through a threeway partnership between Carnegie Mellon University (CMU), Microsoft Research India (MSRI), and the Mathru School for the Blind. Students from CMU, through a tenweek internship with MSRI, conducted a needs assessment and explored a variety of options for developing technology solutions to enhance education for the visually impaired. The students also tested improvements to the BWT by conducting field tests at the original test site for the BWT work; the Mathru School. In Qatar, we partnered with the Al-Noor Institute, a school for the visually impaired. The main goal of our discussions with the Al-Noor Institute was to examine the relevance of the BWT to visually impaired students in Qatar and identify how we could enhance the BWT to better meet their needs. Finally, a partnership with the Sefula School for the Visually Impaired in Mongu, Zambia, was facilitated through the non-profit organization ProjectEducate. We visited the Sefula School over two and a half weeks to train teachers to use the BWT and to get their feedback on how it can be improved for local relevance.

\section{B. Customizability}

In order to field test the BWT in multiple locations, it was necessary to be able to customize the BWT program to cater to the local context. Some of the key features to customize are language, accent, feel, and functionality. In terms of language, the BWT software was designed to easily accommodate adding new character maps for any language which has less than $2^{6}$ characters. To address the issue of local accents, the BWT software uses pre-recorded sound files for all instructions and feedback. The sound files are loaded from an external directory where each of the files is named according to a standard naming convention. This allows us to easily record a new set of sounds (one file for each character in the alphabet, plus a few more phrases used for instruction and feedback) in the local accent, and simply replace the existing sounds without any need to recompile the code. Since tactile interactions are vital to the successful use of the BWT, we were also interested in learning how different it felt to use the stylus with the BWT compared to a slate and stylus used in the local context. We based the design of the E-slate on the dimensions of a standardized slate and used a regular stylus. Finally, we wanted to explore the different needs in terms of functionality required in different schools and countries. To prepare for the possibility of adding new functionality, the BWT software was created in a modular format.

\section{Motivation}

A final enhancement to the BWT was to improve its motivational ability by developing educational games that could be played using the tutor. Educational computer games have been gaining rapid acclaim as motivational tools for children and youth [11]. They can assist people, expand concepts, and teach learners subjects as they play. Formally, one can think of games as a systematic study of the relationship between rules, choices and outcomes in competitive situations [12]. Despite the large number of existing games, we did not find games that are catered to motivate visually impaired children to learn how to write braille. Hence, we developed two games to fill this need.

We collected ideas from teachers at three partner schools to understand the process of learning braille. For example, it was evident at one of the schools that many students struggled with writing braille, but even older students who understood the concepts of braille often made mistakes because of a lack of adequate practice and comfort level with writing braille. Braille writing is an essential skill for a 
blind student to take notes and participate in written exams in some schools. Textbooks in braille are not available for all subjects and are more expensive and inaccessible compared to typical printed books. Therefore, in lowresource communities teachers often dictate notes and students have to take quick and accurate notes. Students then reference these notes to prepare for exams. If a student does not have a solid foundation in braille writing it is hard for them to keep up and study for exams. Also in the higher standards, teacher's lessons are focused on preparing students for state board exams and they do not have time to reinforce braille writing. Practicing writing braille is necessary to develop speed and accuracy. Adding educational games to the braille tutor would encourage students to learn while having fun.

By observing students learn to write braille we found that there were 3 significant steps involved in this learning process. First, a student needs to understand the 6-dot concept. Second, a student needs to learn the dot patterns for each braille symbol. Third, the student has to gain comfort using the stylus and gain control and confidence with embossing the correct dots. The previous version of the BWT had curriculum to teach all of these steps to students but no games to reinforce these steps in a more enjoyable manner. Our goal was to create software for the tutor that would emulate these steps and track and reinforce student weaknesses using the knowledge tracer algorithm.Our primary motivation for incorporating games into the tutor was that games would provide extra incentive to practice braille writing. We were also interested in testing whether material from other subjects could be incorporated into games so multiple subjects could be reinforced simultaneously. Next we describe two games designed for the BWT.

\section{1) Animal Game}

Teachers at our partner school in India informed us that many of the younger students enjoyed learning about animals. Hence, we created a game that would make students identify and spell the name of an animal that made the distinct sound that they heard through the computer. The game was created to help improve spelling and braille writing, and to test whether we could expand the scope of the BWT to teach subjects beyond braille. Using the knowledge tracer, the game identifies the level of the student. Based on the level of the student the software chooses an animal out of a library where animals are categorized by length of the name. Once an animal sound is played, students are prompted to spell the name of the animal. A student is allowed 3 mistakes, with the sound replaying after each mistake, before the answer is revealed. Once the answer is revealed, students are asked to spell the solution. The student's progress is tracked and the software can help the student to practice letter combinations if repeated mistakes are detected on the same letter.

\section{2) Hangman-Inspired Game}

Another goal when developing these games was to increase relevance of the BWT to older students. To accomplish this goal we decided to create a hangmaninspired game for the BWT. This game emulates the traditional word game where a student must guess letters in a word until they know the complete word. In addition to spelling, the game helps students learn how to logically think about general trends found in words while simultaneously expanding their vocabulary. The software is linked to the knowledge tracer module and selects a word length based on a student's level. The software informs the student of the number of letters and asks the student to guess a letter. After each guess, each letter in the word is revealed as a dash or a letter; dashes for letters the student hasn't guessed and letters where the student has guessed correctly. Students must guess the word before making 8 mistakes.

\section{FEEDBACK, OBSERVATIONS, AND DISCUSSION}

Having described the enhancements made to the BWT, we now report and discuss the feedback we received during the field tests and the lessons learned through this feedback and through our observations during the field tests.

\section{A. Additional Feedback}

Through our discussions with the different schools in India, Qatar, and Zambia, we were able to better understand the resources and constraints that teachers of visually impaired children work with on a daily basis. We also learned from the teachers their current methods for teaching braille, any games they use in the classroom, and their curriculum for teaching children to write braille. The need for low-cost tools such as the BWT for assisting these teachers and children was highly evident; especially in under-resourced schools, and in schools where students had multiple disabilities and thus required more individual attention. The enlarged braille cell buttons were very popular in all three schools because they allowed students with less-developed motor skills to learn the concept of the 6 dots. However some teachers found the buttons on the BWT to be too hard and uncomfortable to use over long durations. They suggested using buttons with a softer surface texture or elevating the buttons further from the circuit board. When interacting with the Al-Noor Institute, we were able to improve the surface of the buttons by padding them with a soft plastic sticker that is easily available in bookstores in Qatar at a very low cost. These stickers are flat at the bottom, rounded at the top, and have a much softer texture that is much more comfortable compared to the hard plastic texture of the 6 buttons. Several teachers were also interested in the possibility of having more than one cell represented as buttons in future versions of the BWT to assist younger students to practice the concept of moving from one cell to the next. We plan to incorporate this feedback into future versions of the BWT. 


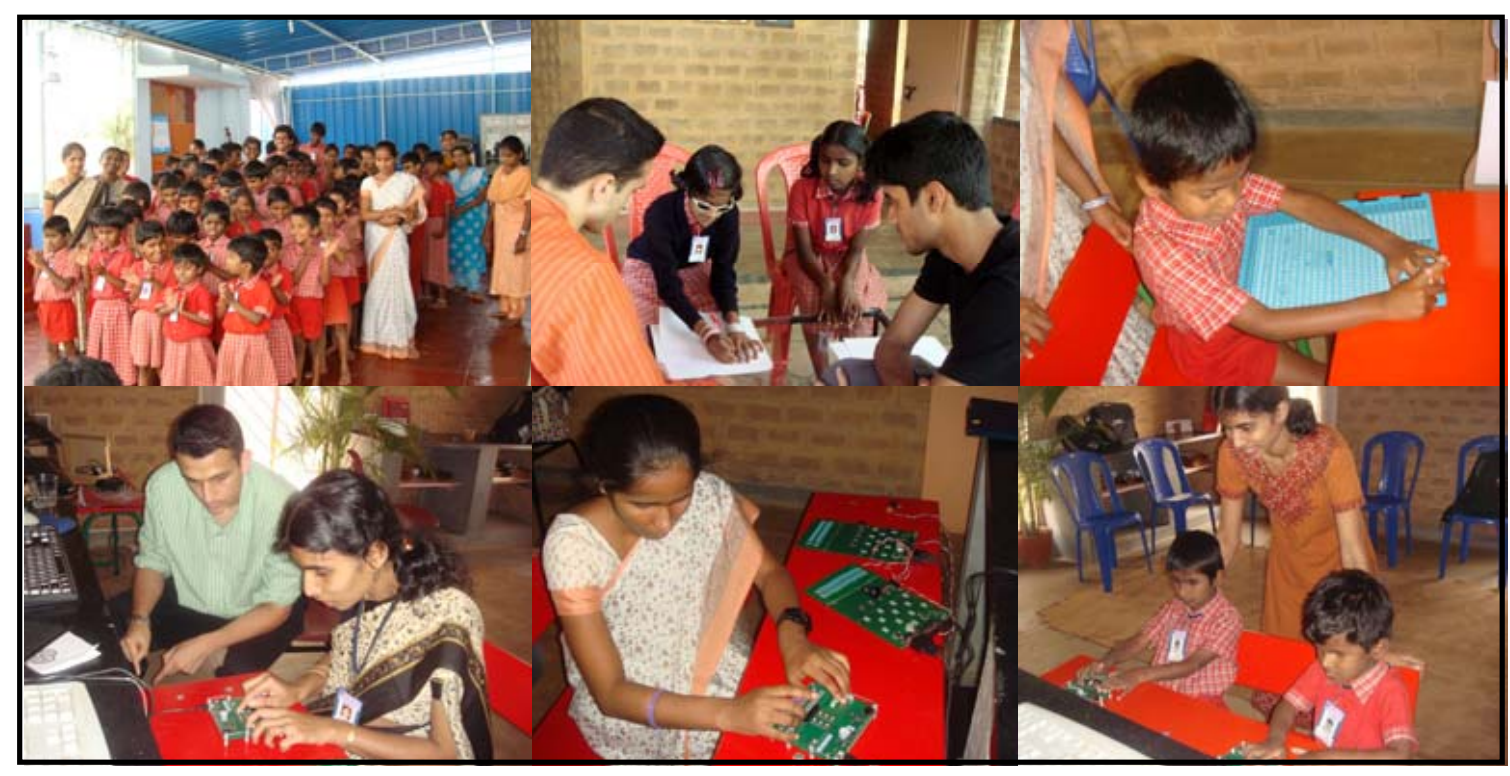

Figure 3: Field Tests at the Mathru School in India

Teachers' time is limited and we found that it was very important to find ways to utilize the BWT to complement classroom instruction time and activities. Learning the 6-dot braille concept is a basic part of the braille curriculum and is something that teachers have told us is difficult for some younger students. To ascertain the effectiveness of the BWT in helping younger students to grasp the basic concept of the 6-dot braille cell, we conducted a field test on a group of 9 students from grades 1 and 2 at the Mathru School. A control group of 9 students of the same age group and level was also setup and this group was not exposed to the BWT. This test was conducted over the course of 5 weeks. Braille instruction was provided 3 times a week in blocks of 2 hour sessions to both groups. Prior to the field test, the teachers were trained on how to operate the BWT effectively. Initially each student was administered a pre-test to measure their knowledge of braille. The pre-test included 6 different elements where the student was asked to: (1) identify all 6 dots in a marble board in the shape of a braille cell; (2) recite the dot patterns of all the letters; (3) make each letter combination using the marble board; (4) press all 6 dots in a braille cell using a stylus; (5) make the letter combinations using a slate and stylus; (6) write a set of 10 words based on Mathru's current spelling lesson of the week. The student was only asked to move to the next step if they successfully completed the previous step. After the completion of 5 weeks of field testing with the BWT all of the students were administered a post-test which was equivalent to the pre-test. Each of the 9 students in the test group showed noticeable improvement and understood the 6-dot concept. This is in contrast to the control group that had only 4 students with noticeable improvement.

The teachers also stated that they were able to more easily identify the concepts that were troubling students in the test group using the BWT, in comparison to the control group.
This demonstrates the diagnostic ability of the BWT which can have a significant impact on learning because teachers can focus on specific skills for children during their classroom instruction. For example, before using the BWT, one student was unable to identify all 6 dots consistently and the teachers did not know why the student was struggling. After working with the BWT, the teacher realized that the student did not know how to find dot 1 . The student would arbitrarily choose any of the dots and consider it to be dot 1 . This diagnosis allowed the teacher to help the student learn the concept of dot 1 , and the student was then able to correctly identify dots 2 through 6 .

Surveying the students on their experience with the BWT also revealed useful feedback. Students preferred to use the BWT compared to the slate and stylus because it allowed them to write letters using the buttons, which is a lot easier than using the stylus. The teachers had to explain to the students that even while using the BWT, the students would eventually have to learn to use the stylus. Students enjoyed receiving the audio feedback from the BWT. The students claimed that they were comfortable with the American English accent but would often remember only the last thing that was said. For example, when the tutor prompted the student to press 1,4 , and 5 , the student would press 5 . After additional practice, the student learned to concentrate more and answer the question correctly. Also, students had memorized the dot patterns for the entire alphabet and could recite the whole set of patterns from A to Z. However, they would often encounter problems when asked for the pattern of a random letter. The BWT was able to identify this problem quickly and therefore is able to assist teachers in customizing educational experiences to meet their students' needs. 


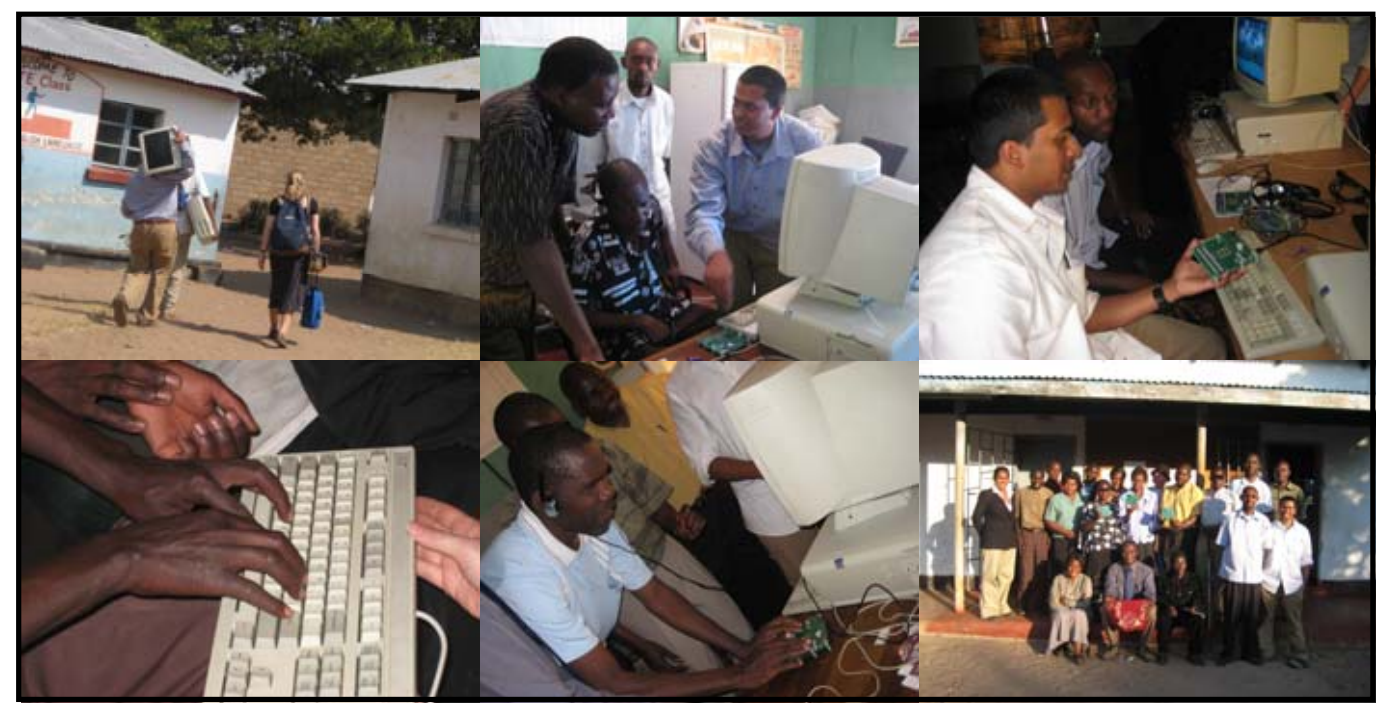

Figure 4: Field Tests at the Sefula School in Zambia

Each student who worked with the BWT at the Mathru School was asked if: (1) they wanted to continue with the BWT, and (2) they liked using the regular slate and stylus better than the BWT. 22 students were tested on the BWT at the Mathru School and all of them agreed with (1) and disagreed with (2). This exercise is not strongly conclusive because some of the questions and answers may have been lost in translation (the teachers translated into local languages), and because many students often give answers they think the interviewer wants to hear. Regardless, observations during the study showed that students enjoyed using the BWT. Future field tests will show if the increased enthusiasm for learning braille can be sustained after the novelty of using the BWT for the first time has passed.

Since the BWT needs to be connected to a computer to function, there were many additional roadblocks that we faced when introducing the device to the Sefula School in Mongu, Zambia. In addition to helping transport and setup the new computers at the school, we had to provide basic training on computer use and maintenance in order to make it possible for the school to use the devices. Overall, we received positive feedback from all three schools and we hope to further improve the BWT based on their feedback.

\section{B. Customizability for Local Context}

The BWT was not useful in Qatar without support for Arabic braille so we decided to enhance the BWT adding support for basic Arabic braille characters. This addition was easily made by simply creating a new character set and incorporating it into the BWT software. The other two schools both taught English braille in the lower grades and hence we did not need to customize for language.

In all three schools we were able to replace the synthesized voice with local voices. The process to replace the sound files proved to be quick and easy and at the Sefula School in Mongu, it only took 30 minutes to record the new sound files using only a laptop and some standard sound recording software. Once the files were recorded and named appropriately, we simply replaced the files on the computer running the BWT program. We got extremely positive feedback on this customization in every location as most of the teachers felt that it would be difficult for their students to understand instructions given by the synthetic voice.

Many teachers and students reported difficulties in using the stylus with some of the BWT devices. We found that these issues were related to bad alignment during assembly of specific devices. Furthermore, there were many different types of styluses and particular types worked much better with the BWT than others. Some teachers reported that it may be difficult for students learning to write braille on the BWT device to transition to using a slate and stylus because, unlike the slate and stylus, the BWT has individual holes for each dot in a slate cell. We plan to make the slate-like cell components of the BWT more similar to the actual slates in future versions.

We got several new requests for functionality in the three test locations. Sighted teachers requested more visual feedback through the BWT software to help them while instructing students. Many teachers were also interested in a more advanced curriculum that incorporated instructions for learning numbers and contractions. During the field tests conducted at the Mathru School, the teachers asked if the BWT could also allow the student to write braille on paper. To address this request, we added a hinged back plate to the BWT below the braille cells. This allowed students to insert paper into the BWT and get a hard copy of what was written, in addition to the audio response on the computer. They were then able to practice reading and writing simultaneously. The teachers welcomed the new feature as it provided them with a way to carry out combined reading and writing exercises. However, additional testing needs to be performed to see if this feature can help in learning to 
read braille and ease the difficulty of learning the mirror effect of reading and writing. Finally, a new feature was added to the software to read aloud the entered text when using the free spelling mode. The reading feature was essential when a student wanted to know what he/she had just written or when a teacher wanted to check on a student's progress.

\section{Motivation through Educational Games}

In India we had the opportunity to test the animal and hangman games with children at the Mathru School. We tested 13 students from grades 4 through 10 on these 2 games. This age group was selected because the games needed players with an intermediate level of vocabulary. Students were tested individually and were first taught the rules of the games. After several sample games where we explained the rules, we allowed the student to play the game and provided help only if needed. All of the students that were tested with the games reported that they enjoyed playing them. The games reinforced braille letter writing and helped students to think in English. Preliminary tests showed that many students have an extremely slow pace of braille writing and games can help them improve their writing speed. The students loved hearing the animal sounds and 10 out of 13 individually surveyed students preferred the animal game to hangman.

During our meetings in Zambia, teachers at the Sefula School were especially interested in incorporating games because they thought the students would respond well to them. They were particularly interested in games that would use music because there is a big emphasis on music performance at the school. They suggested that different musical tones could be used to indicate which dot in the braille cell the student pressed. There were also suggestions to provide a "score" that would give students an idea of what their progress was as they used the device. Observations at the Al Noor Institute in Qatar showed us that the Dominos game is very popular among visually impaired students and hence we were able to design a similar game that can be played on the BWT. Overall, we learned that games can require more customization to the local culture to improve their popularity among students.

\section{CONCLUSIONS AND FUTURE WORK}

Braille literacy is an important skill that significantly enhances the opportunities for visually impaired individuals to play a meaningful role in modern society. Our research advances the state-of-the-art in assistive technology for the blind by enhancing a low-cost automated tutor designed to teach braille writing skills to children. The outcome of this work is an enhanced relatively low-cost tool for increasing braille literacy in visually impaired communities around the world. Future work includes designing additional games and musical elements to the BWT, extending the field testing to different locations and for longer durations, adding other languages and mathematic capabilities, improving the adaptive feedback, and making a stand-alone BWT that does not require connection to an external computer.

\section{ACKNOWLEDGMENT}

The authors wish to acknowledge all of the contributions of students and faculty who have worked on this project in the past, and the many teachers and students from the Mathru School for the Blind in India, Al-Noor Institute for the Blind in Qatar, and Sefula School for the Visually Impaired in Zambia who participated in our field studies. Special thanks are due to Nidhi Kalra, Tom Lauwers, Tom Stepleton, and Daniel Dewey for designing and implementing a large part of the current hardware and software for the BWT. We are also grateful to Microsoft Research India and ProjectEducate for their help with facilitating the field studies in India and Zambia. Finally, we acknowledge the ongoing work of Imran Fanaswala, Wael El-Ghazzawi, Ameer Abdul Salam, for improving the BWT.

\section{REFERENCES}

[1] Nidhi Kalra, Tom Lauwers, and M. Bernardine Dias, "A Braille Writing Tutor to Combat Illiteracy in Developing Communities," Artificial Intelligence in Information Communication Technology for Development workshop at IJCAI 2007. Available at: http://www.ri.cmu.edu/pub_files/pub4/kalra_nidhi_2007_1/kalra_nidh i_2007_1.pdf.

[2] International Council for Education of People With Visual Impairment, "EFA-VI Global Campaign - A Shared Challenge." Available at: http://www.icevi.org/efa/global_campaign\%20.html

[3] World Health Organization, "Fact sheet 282: Visual impairment and blindness," World Health Organization, May 2009. Available at: http://www.who.int/mediacentre/factsheets/fs282/en/index.html.

[4] Perkins School for the Blind, Perkins Products online store. Available at: http://support.perkins.org/PerkinsProducts.

[5] Nidhi Kalra, Tom Lauwers, D. Dewey, Tom Stepleton, and M. B. Dias, Iterative Design of A Braille Writing Tutor to Combat Illiteracy, IEEE/ACM International Conference on Information and Communication Technologies and Development (ICTD), 2007. Available at: http://www.ri.cmu.edu/pub_files/pub4/kalra nidhi 2007 2/kalra nidhi 2007 2.pdf.

[6] Application of AI in Education. Joseph Beck, Mia Stern and Erik Haugsiaa 7 September, 2007. Available at: http://www.acm.org/crossroads/xrds3-1/aied.html

[7] Burns Tom \& Sinfield Sandra. Teaching, Learning \& Study Skills. SAGE Publications Inc, 2455 Teller Road, Thousand Oaks, California, 2004.

[8] Interview with Professor Yasser Mohammed Al-Shafai, Arabic Teacher at Al-Noor Institute.

[9] Conati Critina, Gertner Abigail, VanLehn Kurt, Druzdel J. Markek. "Online Student Modeling for Coached Problem Solving Using Bayesian Networks". University of Pittsburgh, PA, U.S.A.

[10] Kay Judy. Stereotypes, Student Models and Scrutability. Basser Dept of Computer Science. University of Sydney.

[11] Chris Davies, Geoff Hayward and Linariah Lukman, "14-19 and digital technologies," Futurelab Series, Report 13, April 2005.

[12] Jonas Heide Smith, "The Games Economists Play - Implications of Economic Game Theory for the Study of Computer Games," Game Studies, December 2006.

[13] TechBridgeWorld Automated Braille Writing Tutor project website: http://www.techbridgeworld.org/brailletutor

[14] Carr, B. and Goldstein, I. 1977. Overlays: a Theory of Modeling for Computer-aided Instruction, Technical Report, AI Lab Memo 406, MIT 